\title{
Learning Styles and Caring Behaviors Among Students in Different Nursing Programs: Implications for Nursing Education
}

\author{
Yuh-Shiow Li*1,3, Wen-Pin Yu², Bao-Huan Yang1,4, Chin-Fang Liu ${ }^{1,5}$ \\ ${ }^{1}$ Department of Nursing, Chang Gung University of Science and Technology, Taiwan \\ ${ }^{2}$ Department of Nursing, Chang Gung Memorial Hospital, Taiwan \\ ${ }^{3}$ Department of Nursing Management, Chang Gung Memorial Hospital, Taiwan \\ ${ }^{4}$ Department of Physical Medicine and Rehabilitation, Chang Gung Memorial Hospital, Taiwan \\ ${ }^{5}$ Department of Nursing, Chang Gung Memorial Hospital, Taiwan
}

Received: 眥: September 22, 2018; Published: 裵 September 28, 2018

*Corresponding author: Yuh-Shiow Li, Associate Professor, Department of Nursing, Chang Gung University of Science and Technology, Address: No.261, Wen-Hua $1^{\text {st }}$ Road, Kweishan, Taoyuan 333-03, Taiwan

Abstract

Purpose: This study was to explore the relationship between the learning styles and caring behaviors of nursing students in a 5-year associate degree nursing program and 4-year and 2-year bachelor of science nursing programs.

Methods: Two measurement instruments were used: the Chinese version of the Myers-Briggs Type Indicator (MBTI) to identify learning styles and the Caring Behavior Scale (CBS) to measure caring behaviors. The research period was from 2015 to 2016. Ethical approval was granted. To analyze the study data, descriptive statistics, one-way analysis of variance and an independent t-test were performed.

Results: Three common learning styles were found: ENFP (extraversion, intuition, feeling, perceiving), IST) (introversion, sensing, thinking, judging) and ISFJ (introversion, sensing, feeling, judging). The results indicated that caring behaviors are significantly related to learning styles. Moreover, there was a significant difference between caring behaviors and subscales extraversion/introversion, respectively.

Conclusion: The information about learning style and caring behavior is a major consideration while planning for successful nursing education. Further research is recommended. This study provides a reference for the design of nursing curricula, as well as the use of varied teaching strategies to appropriately respond to students' needs, so that they can mitigate problematic learning situations and provide quality of care in the workplace.

Keywords: Associate Degree of Nursing; Bachelor of Science in Nursing; Caring Behaviors; Learning Styles

\section{Introduction}

The goal of nursing education is a commitment to prepare competent and caring nurses professionals to provide quality care that promotes the health of human beings. Caring is the basic core concept of the nursing profession [1] and nursing educators need to consider that individuals have different learning styles in the educational process. Learning style is a crucial factor that influences individuals to learn efficiently and effectively [2]. In addition, nursing attrition is an important issue to be solved in the worldwide nursing workforce [3], as it is a challenge for institutions of nursing education to produce qualified nurses; therefore, nursing faculties must be concerned with nurturing nursing novices to become experts in providing qualified care.

Nursing is caring and requires nursing students to learn how to become professional nurses in clinical settings. Nursing students should be regarded as clients in health facilities and show the necessary care and concern. In addition, research indicates that information regarding learning styles can be helpful in designing nursing curriculums that provide the best learning outcomes for students in the nursing profession [4]. However, there is a lack of studies regarding the learning styles and caring behaviors of nursing students. As a result, little is known about how ethnicity affects learning style preferences. Therefore, the purpose of this study is to identify the relationship between the learning styles and caring behaviors of students in different nursing programs.

\section{Nursing Education System in Taiwan}

There are two pathways to enter the nursing profession in Taiwan: the technical and vocational education system and the general education system (Figure 1). Junior high school graduates can advance to senior high school and then, further their education in college or university. This general education trend provides the base for generic nursing programs at university/college and graduate levels, including master and doctoral degrees. The other path is the technical and vocational education system. There are three different levels of nursing education available in the 
technical and vocational education system. The first is to enroll in an Associate Degree Nursing (ADN) program in a five-year junior college and receive an associate degree after completing the program. The second is to study at a university/college and receive a Baccalaureate Science Degree in Nursing (BSN). The third way is to complete a nursing graduate program that offers master and doctoral degrees.

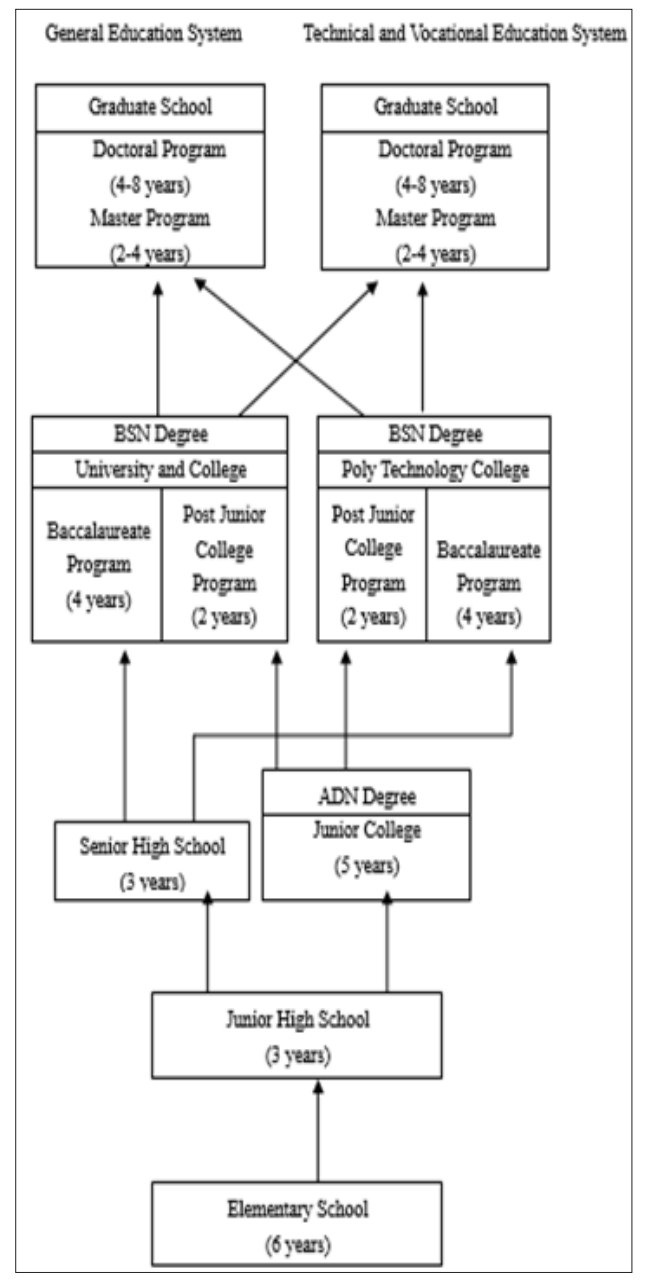

Figure 1: Current nursing education system in Taiwan.

\section{Learning Styles and the Myers-Briggs Type Indicator}

Understanding individual learning styles is helpful both for learners to take appropriate measures to learn effectively and efficiently and for educators to moderate their teaching contents and adopt more appropriate teaching strategies. Numerous definitions of learning styles have been proposed. Some researchers suggested that a learning style is related to how learners perceive, interact with and respond to the learning environment [5]. Other researchers indicated that learning styles are the ways that different individuals characteristically approach different learning tasks [4]. Awareness of personal learning styles can benefit students in many ways, meaning students can be more confident in their strengths and develop diverse strategies for coping with the challenging situations that inevitably arise.

The Myers-Briggs Type Indicator (MBTI) provides abundant information, which is valuable in understanding and gaining insight into individuals' learning processes. The MBTI has implications in academia, education and career counselling and is based on Carl Jung's theory [6], which includes four scales of dichotomies: Extraversion/Introversion (E/I), Sensing/Intuition (S/N), Thinking/Feeling (T/F) and Judging/Perceiving (J/P). The MBTI identifies individual differences in learning styles and provides a rational structure for designing activities for learners that encourage learning, regardless of their preferred learning style. The "Jungian model suggests that people will be at their best when they have effective command of their dominant function" [7]. In addition, in the sample of nursing students, research indicates that the predominant subjects are sensing and judging [8,9].

\section{Caring in Nursing and Relative Research}

Caring is the heart of nursing, which requires knowledge, competency and interpersonal skills to provide care for patients and includes conformance to the ethical and legal principles of care [10]. Researchers have indicated that "carers" are faculty; while the "ones cared for" are students. Student-faculty interactions are constructed in a sociocultural context, as the social space where student-faculty interactions occur may influence the perceptions of caring, as based on Watson's 10 carative factors [11]. Ayala \& Calvo [12] stated that the essence of caring behavior is determined by cultural differences. Caring behaviors vary with cultural backgrounds and traditions [13]. Consequently, caring is a distinguished element in the field of nursing.

In Chinese culture, nursing students are taught to treat patients kindly during the educational training process, as patients are similar to their families [1]. A Confucian value present in Chinese culture is to be in harmony with surrounding social contexts [1]. Confucianism states the value of both strong family bonds and educational impact in Chinese culture. Caring is demonstrated by a nurse's professional behavior, which encompasses a range of affective and instrumental skills. There is a significant relationship between students' clinical skills and their ability to apply knowledge and understand the concept of patient care [14].

\section{Learning Styles and Caring Behaviors}

Faucett et al. [15] measured moral reasoning, which showed that the moral reasoning scores of those who were Introverts were higher than those of Extroverted subjects. Intuitive subjects were higher than Sensing types and Perceptive people were higher than Judgers. Thinking and Feeling people were not significantly different. Moreover, a caring voice when making ethical decisions correlated positively with the Feeling dimension of the MBTI [16]. Jenkins et al. [17] found a positive relationship between empathy and the Feeling dimension of the MBTI and noted that Feeling individuals are sympathetic, understanding of others and attentive to others' values and that those same qualities are essential for an empathetic response.

The typical beginning nursing student favours a learning environment characterized by caring relationships, structure, permission to be assertive, utilizes nurturing behaviors, uses practical materials that relate to concrete realities and learning activities that require touch, muscular sensations and kinesthetic senses [8]. The application of learning style demands that 
administrators and educators become more active participants in educational enterprises by providing a caring and supportive learning environment in which students can play a major role in their own learning [4], while engaging in classroom learning and clinical settings that enrich their lives and enhance their potential success in a nursing career.

\section{Methods}

This study employed a descriptive and exploratory design, where nursing students were asked to complete the Chinese version of the MBTI (From M) questionnaire, in order to identify their learning styles and the Caring Behaviors Scale (CBS), in order to assess the subjects' caring behaviors towards patients. Participants also completed a one-page demographic sheet. The investigators utilized measures with quantifiable coding of the operationalise variables and statistical procedure to analyse the data collected.

\section{Sample}

The study sample of nursing students was selected by convenience sampling of a class section of approximately 50 nursing students from three programs, including the five-year ADN program, the two-year and four-year baccalaureate degree of nursing programs, at an institute located in the north of Taiwan. A total of 792 nursing students participated in this study. Missing data included 12 subjects, who either failed to complete the questionnaire or did not participate in the survey completely. The participation rate for completed questionnaires was $98 \%$.

\section{Instrumentation}

The traditional Chinese version of the MBTI (Form M) is an instrument designed for identifying student learning styles, which was published in 2003 and participants completed the MBTI questionnaire in 15-25 minutes by using forced-choice items. Although the MBTI questionnaire was originally developed in the U. S., it is a widely utilized psychological instrument that has been translated internationally into more than 30 languages and applied in many countries, including China, Canada, Britain and Australia $[6,7]$. The MBTI determines four dichotomous dimensions of Introversion-Extraversion, Sensing-Intuition, Thinking-Feeling and Judging-Perceiving, as based on Jung's [18] type theory. Each individual measured by the MBTI instrument receives a four-letter code type from a possible 16 combinations, with each letter of the code representing the learning preference of one dimension. The researchers administering the instrument also scored the MBTI questionnaire.

The other instrument is the Caring Behaviors Scale (CBS), which was designed to assess participants' caring behaviors in this study. The CBS, as developed by Ou and Lin [19], contains 28 items and includes three dimensions: (1) Helping the patient through the illness trajectory; (2) Advocating for the patient; and (3) Knowing the patient. Each dimension consists of 9 or 10 items. An item pool for the Caring Behaviors Scale (CBS) was developed from in-depth individual interviews and focus group interviews of 11 Taiwanese nursing faculty members, 3 clinical specialists and a senior student focus group. However, regarding the 56-item CBS, this research used the split-half method to avoid the carry-over effect for decreasing bias in collecting data. Consequently, there are 28 items in total in the short form of the CBS. Previous research supported that the CBS is a reliable and valid instrument $[20,21]$.

\section{Reliability and Validity}

The MBTI is a good instrument, which has high reliability and validity. Test-retest reliability was applied for the MBTI (Form M) questionnaire, which ranged from .87 to .93 in a sample of 258 at the Public Utilities Company [6]. The reliability of Form was $0.89-0.94$ in a national sample of 3,036 participants [7], while the coefficient alpha ranged from 0.91 to 0.92 in another national sample of 2,859 participants [6]. Researchers also supported that the internal consistency of the four MBTI scales is high and that the test-retest reliability of the MBTI shows consistency over time [6]. In addition, the MBTI (Form M) was ranked most accurate by $58-78 \%$ of the participants [22]. Researchers indicated that a confirmatory factor analysis of Form M found a four-factor model with a 0.95 adjusted goodness-of-fit based on 3,036 international participants [7]. Huszczo and Endres [23] stated high reliability and validity for the MBTI instrument. Previous research supported that the Chinese version of the MBTI has high content validity, criterion referent validity and construct validity $[24,25]$. Above all, the MBTI's Form $\mathrm{M}$ is a highly reliable and valid instrument. The CBS includes 28 items to measure the participants' caring behaviors using a 4-point Likert scale ( 0 = Never, 1 = Sometimes, 2 = Often, 3 = Always).

The CBS total score, including three subscales, is from 0 to 84 and each subscale ranges from $0-30$. A high CBS score indicates that more caring behavior was presented in this study. The Cronbach's alpha coefficient of the CBS was 0.94 and the test-retest reliability coefficient was 0.86 [26]. Moreover, the CBS's subscales coefficient alphas ranged from $0.83-0.87$ on the three subscales (helping the patient through the illness trajectory, $r=0.83$; advocating for the patient, $r=0.86$ and knowing the patient, $r=0.87$ ). The testretest reliability coefficients were $0.82,0.80$ and 0.82 on the three dimensions, respectively [1]. The CBS represented good reliability and had acceptable results [25]. In addition, the validity of CBS was determined by six nursing experts, which resulted in 0.82 of the content validity index [26]. The factor analysis approach was used to evidence the content validity and construct validity of the CBS and the analysis of variance was $67.4 \%$ in the previous research [19]. Consequently, researchers have employed the CBS on nursing students in both the baccalaureate and associate degree programs and both have proven that the CBS instrument has strong reliability and acceptable validity [24,26].

\section{Ethical Considerations}

The institutional research board of the participating institute approved this study. The nursing students received a formal explanation before administering the questionnaires and those who agreed to participate signed a consent form. Participants were informed about their rights, such as confidentiality of personal data and free withdrawal from the study at any point without justification or consequences. The investigators administered and scored the MBTI and CBS questionnaires. The nursing students voluntarily participated in this study and all data were confidential and anonymous. 


\section{Data Analysis}

After completing the MBTI and CBS instruments, the questionnaires were scored by the researchers. Data were analyzed using the Statistical Package for the Social Science (SPSS 21.0) for descriptive statistics, including frequencies, percentages, ranges, means, standard deviations and modes. Study data in all analyses was evaluated with an alpha level of .05. This study aimed to explore the relationship between learning styles and caring behaviors in Taiwanese nursing students, thus, descriptive statistics, one-way analysis of variance and Pearson's correlation coefficient were performed in this study.

\section{Results}

The sample of this study was a total of 792 nursing students; 201 students in the five-year ADN program, 184 students in the four-year BSN program and 395 students in the two-year BSN program (178 students in the day program and 217 students in the evening program). There were only 21 male participants in this study and the questionnaire completion rate was $98 \%(n=780)$. Missing data included 12 nursing students, who failed to finish the CBS and NSC questionnaires. Table 1 presents the demographic data of the participants. The age of participants ranged from 17 to 46 years old. The majority of nursing students was $20-21$ years $(n=462$, $59.3 \%$ ). The average age of participants was 21 years. The majority of nursing students was unmarried ( $\mathrm{n}=754,96.7 \%)$. There were 576 participants $(73.8 \%)$ with no previous experience in nursing work. A significant relationship between learning styles and caring behaviors was detected using one-way analysis of variance ( $p$ $=0.001, \mathrm{df}=15$ ).

Table 1: Participants' demographic profiles.

\begin{tabular}{|c|c|c|}
\hline Characteristic & $n$ & $\%$ \\
\hline \multicolumn{3}{|c|}{ Gender } \\
\hline Female & 759 & 97.3 \\
\hline Male & 21 & 2.7 \\
\hline \multicolumn{3}{|c|}{ Age (years) } \\
\hline 17 & 15 & 1.9 \\
\hline 18 & 95 & 12.2 \\
\hline 19 & 89 & 11.4 \\
\hline 20 & 204 & 26.2 \\
\hline 21 & 258 & 33.1 \\
\hline 22 & 42 & 5.4 \\
\hline 23 & 21 & 2.7 \\
\hline 24 & 12 & 1.5 \\
\hline 25 & 3 & 0.4 \\
\hline $26-30$ & 13 & 1.7 \\
\hline $31-35$ & 13 & 1.7 \\
\hline $36-40$ & 8 & 1 \\
\hline $41-46$ & 7 & 0.9 \\
\hline \multicolumn{3}{|c|}{ Marital status } \\
\hline Single & 754 & 96.7 \\
\hline
\end{tabular}

\begin{tabular}{|c|c|c|}
\hline Married & 25 & 3.2 \\
\hline Divorced & 1 & 0.1 \\
\hline \multicolumn{3}{|c|}{ Nursing work experience (years) } \\
\hline None & 576 & 73.8 \\
\hline$<1$ & 114 & 14.6 \\
\hline $1-5$ & 55 & 7.1 \\
\hline$>5$ & 35 & 4.5 \\
\hline Missing & 12 & \\
\hline
\end{tabular}

Note: $\mathrm{N}=792$. Missing data included 12 participants who did not finish the questionnaires completely.

The predominant learning styles were ENFP, ISTJ and ISFJ for the total sample of 792 nursing participants in the five-year ADN program, the four-year and the two-year BSN programs in this study (Table 2). Pairs and temperaments are usually used in MBTI research to demonstrate how the combinations of any 2 letters among the16 types are manifested by the nursing participants. As listed in Table 3, the predominant pairs and temperaments of the Taiwanese nursing students were NF (34.1\%), EN (32.7\%) and SJ (32.1\%). Less frequent were SP (11.7\%), TP (15.5\%) and ES (17.8\%).

Table 2: Frequency distribution of the types among the participating nursing students.

\begin{tabular}{|c|c|c|c|}
\hline Type & Frequency & Percent & $\begin{array}{c}\text { Cumulative } \\
\text { percent }\end{array}$ \\
\hline ENFJ & 69 & 8.7 & 8.7 \\
\hline ENFP & 94 & 11.9 & 20.6 \\
\hline ENTJ & 48 & 6.1 & 26.7 \\
\hline ENTP & 44 & 5.6 & 32.3 \\
\hline ESFJ & 42 & 5.3 & 37.6 \\
\hline ESFP & 21 & 2.7 & 40.3 \\
\hline ESTJ & 62 & 7.8 & 48.1 \\
\hline ESTP & 14 & 1.8 & 49.9 \\
\hline INFJ & 56 & 7.1 & 57 \\
\hline INFP & 47 & 5.9 & 62.9 \\
\hline INTJ & 39 & 4.9 & 67.8 \\
\hline INTP & 42 & 5.3 & 73.1 \\
\hline ISFJ & 70 & 8.8 & 81.9 \\
\hline ISFP & 35 & 4.4 & 86.3 \\
\hline ISTJ & 76 & 9.6 & 95.9 \\
\hline ISTP & 21 & 2.7 & 98.6 \\
\hline Missing & 12 & 1.5 & 100 \\
\hline Total & 792 & 100 & \\
\hline
\end{tabular}

Note: N=792. "Missing" means that the subjects either did not finish the MBTI and CBS questionnaires or did not participate in the study completely. Data in bold font represents the highest percentage among the participating students. 
Table 3: Pairs and temperaments of the participating nursing students.

\begin{tabular}{|c|c|c|}
\hline Type & Frequency & Percent \\
\hline IJ & 241 & 30.9 \\
\hline IP & 145 & 18.6 \\
\hline EP & 173 & 22.2 \\
\hline EJ & 221 & 28.3 \\
\hline ST & 173 & 22.2 \\
\hline SF & 168 & 21.5 \\
\hline $\mathrm{NF}$ & 266 & 34.1 \\
\hline NT & 173 & 22.2 \\
\hline SJ & 250 & 32.1 \\
\hline SP & 91 & 11.7 \\
\hline NP & 227 & 29.1 \\
\hline $\mathrm{NJ}$ & 212 & 27.2 \\
\hline $\mathrm{TJ}$ & 225 & 28.8 \\
\hline $\mathrm{TP}$ & 121 & 15.5 \\
\hline FP & 197 & 25.3 \\
\hline $\mathrm{FJ}$ & 237 & 30.4 \\
\hline IN & 184 & 23.6 \\
\hline EN & 255 & 32.7 \\
\hline IS & 202 & 25.9 \\
\hline ES & 139 & 17.8 \\
\hline ET & 168 & 21.5 \\
\hline $\mathrm{EF}$ & 226 & 29 \\
\hline IF & 208 & 26.7 \\
\hline IT & 178 & 22.8 \\
\hline
\end{tabular}

Note: Data in bold font represents the highest percentage among the participating students.

Table 4 describes MBTI preferences and CBS scores and shows that, according to the cumulative averages, nursing students with extrovert, intuition, thinking and judging preferences had higher scores than those with introvert, sensing, feeling and perceiving preferences. There was a significant difference between MBTI types and the score of CBS ( $p=0.001)$. As can be seen in Table 4, independent t-testing revealed significant relationships between stress scores and the subscales E/I $(p<0.001)$. Table 5 shows the distribution of the CBSC scores among the different MBTI types. Overall, nursing participants with ENFJ, ISTP and ESTP preferences had the highest averages of the CBS scores in this study.

Table 4: Participating students' CBS scores and MBTI types.

\begin{tabular}{|c|c|c|c|c|c|c|}
\hline \multirow{2}{*}{ Type } & \multirow{2}{*}{ N } & \multirow{2}{*}{ Percent } & \multirow{2}{*}{ Mean } & \multirow{2}{*}{ SD } & \multicolumn{2}{|c|}{ CBS } \\
\cline { 6 - 7 } & & & & & Minimum & Maximum \\
\hline E $^{*}$ & $\mathbf{3 9 4}$ & $\mathbf{5 0 . 5}$ & $\mathbf{5 7 . 6 5}$ & 12.17 & 24 & 84 \\
\hline $\mathrm{I}^{*}$ & 386 & 49.5 & 54.45 & 11.82 & 18 & 78 \\
\hline $\mathrm{S}$ & 341 & 43.7 & 55.12 & 11.95 & 18 & 84 \\
\hline $\mathrm{N}$ & $\mathbf{4 3 9}$ & $\mathbf{5 6 . 3}$ & $\mathbf{5 6 . 8}$ & 12.17 & 20 & 84 \\
\hline $\mathrm{T}$ & 346 & 44.4 & $\mathbf{5 6 . 3 3}$ & 11.75 & 24 & 84 \\
\hline
\end{tabular}

\begin{tabular}{|c|l|l|l|l|l|l|}
\hline F & $\mathbf{4 3 4}$ & $\mathbf{5 5 . 6}$ & 55.85 & 12.37 & 18 & 84 \\
\hline J & $\mathbf{4 6 2}$ & $\mathbf{5 9 . 2}$ & $\mathbf{5 6 . 1 9}$ & 11.58 & 24 & 84 \\
\hline P & 318 & 40.8 & 55.88 & 12.83 & 18 & 84 \\
\hline
\end{tabular}

Note: $\mathrm{N}=792$. Missing data included 12 participants who did not finish the MBTI questionnaire completely. *There was a significant difference between the CBS score and the subscales E/I ( $p<0.001$, respectively).

Table 5: Distribution of CBS scores among different MBTI types.

\begin{tabular}{|c|c|c|c|c|c|}
\hline \multirow{2}{*}{ Type } & \multirow{2}{*}{$\boldsymbol{N}$} & \multirow{2}{*}{ Mean } & \multirow{2}{*}{$\boldsymbol{S D}$} & \multicolumn{2}{|c|}{ CBS } \\
\cline { 5 - 6 } & & & & Minimum & Maximum \\
\hline ESTJ & 62 & 57.19 & 12.39 & 30 & 79 \\
\hline ESTP & 14 & $\mathbf{6 0 . 1 4}$ & 12.17 & 42 & 84 \\
\hline ESFJ & 42 & 56.07 & 10.27 & 35 & 75 \\
\hline ESFP & 21 & 53.38 & 14.98 & 28 & 81 \\
\hline ENTJ & 48 & 56.27 & 12.87 & 24 & 77 \\
\hline ENTP & 44 & 57.27 & 12.86 & 28 & 80 \\
\hline ENFJ & 69 & $\mathbf{6 1 . 5 4}$ & 11.5 & 39 & 84 \\
\hline ENFP & 94 & 57.28 & 11.57 & 35 & 84 \\
\hline ISTJ & 76 & 54.11 & 9.76 & 35 & 77 \\
\hline ISTP & 21 & $\mathbf{6 1 . 5 2}$ & 11.15 & 47 & 78 \\
\hline ISFJ & 70 & 52.91 & 11.89 & 28 & 76 \\
\hline ISFP & 35 & 52.14 & 14.12 & 18 & 78 \\
\hline INTJ & 39 & 57.1 & 9.95 & 35 & 75 \\
\hline INTP & 42 & 53.6 & 12.61 & 25 & 75 \\
\hline INFJ & 56 & 54.8 & 11.62 & 27 & 75 \\
\hline 1INFP & 47 & & & & 76 \\
\hline Missing & 12 & 53.96 & 13.35 & 20 & 74 \\
\hline Total & 780 & 56.07 & 12.1 & 18 & 76 \\
\hline
\end{tabular}

Note: Bold font represents the highest number among the participant students.

\section{Discussion}

The majority of nursing students in Taiwan are female. In this study, the predominant learning styles were ENFP, ISTJ and ISFJ. The findings of the current study agrees with those of previous research, in that students in a nursing major tend to have sensing and judging preferences to a greater degree than in non-nursing majors [9,27]; therefore, nurses are often classified as combined sensing and judging preference. In other words, nurses tend to focus their main interest on facts, as well as establishing their reality using one or more of the five senses in a pragmatic style. Moreover, in their judgments, these individuals prefer to schedule their time carefully through structure and planning and tend to be decisive and committed to organizing their activities [6]. A significant relationship between caring behaviors and different nursing programs was detected. The averages of caring behaviors in different nursing programs were as follows: five-year program 58.06 ( $\mathrm{n}=201$ ), four-year program 53.46 ( $\mathrm{n}=184)$, two-year program (day program) $56.44(\mathrm{n}=178)$ and two-year program (evening program) $56.12(\mathrm{n}=217)$. The average of the CBS scores 
among the 780 participating nursing students was 56.07 (The score of caring behaviors ranged from 0 to 84 ).

The finding was similar to the previous researchers' study [19], the standardized CBS score was 59.55, which located at the medium degree of the total of 287 Taiwanese participants in a 5-year associate degree of nursing program. In this study, the highest CBS score was $58.06(n=201)$ in a 5 -year ADN program in the total sample of 792 nursing students. The reasons include some considerations, such that nursing students in the ADN program are younger, therefore, they receive the educational training about the caring concept are earlier than others. Consequently, the highest average of the CBS scores was in the ADN program, due to students' great recognition in caring. There was a significant relationship $(p=0.001)$ between learning styles and caring behaviors and significant difference between the CBS score and the subscales E/I $(\mathrm{p}<0.001)$. This relationship may be the result of individuals with extraversion having a preference in the current study for being more caring clients. The findings revealed that extraverts may seek to be energized by activities and interactions; therefore, those who tend towards extraversion seek to interact with others by providing caring behaviors.

These findings support previous studies, in that individual traits may be used for specific predictions or explanations [6]. However, there was no significant correlation between caring behaviors and the Thinking/Feeling scale of MBTI. This finding is not congruent with other studies, which found a relationship between caring as a moral voice and the Feeling preference $[16,17]$. The explanations for this unexpected finding may be influenced by several factors, including the homogeneity of the sample, scoring of the instruments, MBTI type and level of preference in this study.

\section{Implications for Nursing Education}

The goal of nursing education is to prepare future nurses to provide quality care for people. This study found three predominant learning styles, ENFP, ISTJ and ISFJ, which nursing educators should be aware of in order that they are presented in classrooms and clinical settings. The findings of this study provide information that can enhance program design, service provision and learning outcomes for nursing students. This information can be used to modify the modes of delivery and participation, student assignments, structuring learning groups and other methods to enhance caring. Educators need to consider the individual in learning, as well as the importance of caring, in order to build an effective curriculum. When utilizing information on learning styles and caring behaviors, it would be important to understand that the differences in 16 types are valuable and necessary and that all MBTI types are needed in the field of health care. Research indicates that a better understanding of learning styles can be beneficial to both teachers and students in providing good quality care. Teachers should be aware of the learning styles present in their classrooms and clinical settings, in order to adjust their presentations accordingly. There are many challenges in nursing education, such as recruitment.

Nursing shortages are a global issue, which is compounded by an aging population, a cultural expectation in Taiwan that young women will leave nursing to marry and care for their family and fewer students being attracted to nursing preparation programs. While the nursing shortage around the world involves more variables than learning style, educators are likely to lose fewer potential nurses if instruction can be improved. The results of this study support increasing faculty understanding of learning styles, as such understanding will be useful to both the students and teachers who will encounter each other in classrooms and clinical settings. An understanding of the learning styles that affect persistence in higher education is vital to improving retention efforts. In addition, caring is the heart of nursing and all people need competent professional nurses to deal with their health status. Nursing educators are shown how to teach students to market their practice, develop an appropriate plan and deal with the issues that affect the advanced practices of nursing. Nursing educators can also learn new strategies and tools to improve their advanced teaching skills. Consequently, nurses have high levels of educational preparation, which may have beneficial impact on the outcomes of patients and enhance the quality of care.

\section{Conclusion}

Researchers indicate that using a learning style approach can empower staff development to create an optimal environment for successful staff learning to assist in providing quality care with increasing retention [28]. As nursing is a discipline in which clinical experience is a very important component, it is possible to increase the types of classroom activities that better match the learning styles of the practical learners. Nursing education faces numerous challenges in preparing future nurses, such as attrition in nursing, professional isolation and lack of support [29]. This study indicates a significant difference between caring behaviors and learning styles. Educators should recognize learning styles as an important element needed to create an optimal learning environment, while increasing retention, in order to provide the best quality of care [4,29].

Limitations of this study include no comparable group of enough male nursing students (only 21 male participants) to study gender differences and one single institute was examined as part of the investigation. In addition, cultural effects may be a factor that influences how people learn and provide quality care [30]. Further studies are recommended to examine an international sample of nursing students in order to generate more comprehensive conclusions. Consequently, this study recommends that educators use effective teaching strategies to address the varying learning styles, thereby, providing holistic care by caring professionals once their nursing careers are established, in order to enhance nursing education.

\section{Acknowledgements}

This study was supported by grants from the Ministry of Science and Technology, Taiwan (Award No. MOST 105-2511S-255-002) and the Chang Gung Medical Foundation (Award No. CDRPF3H0011). The authors thank Dr. Pay-Fan Lin for her permission to use the data collection instrument in completing the study. We also thank all colleagues and students who kindly participated in this study. 


\section{References}

1. Li Y, Yu W, Yang B, Liu C (2016) A comparison of the caring behaviours of nursing students and registered nurses: implications for nursing education. J Clin Nurs 25(21-22): 3317-3325.

2. Anderson I (2016) Identifying different learning styles to enhance the learning experience. Nurs Stand 31(7): 53-63.

3. Feng D, Su S, Yang Y, Xia J, Su, Y (2017) Job satisfaction mediates subjective social status and turnover intention among Chinese nurses. Nurs Health Sci 19(3): 388-392.

4. Li Y, Yu WP, Liu CF, Shieh SH, Yang BH (2014) An exploratory study of the relationship between learning styles and academic performance among students in different nursing programs. Contemp Nurse 48(2): 229-239.

5. Gonzales LK, Glaser D, Howland L, Clark MJ, Hutchins S, et al. (2017) Assessing learning styles of graduate entry nursing students as a classroom research activity: A quantitative research study. Nurse Educ Today 48: 55-61.

6. Quenk NL (2009) Essentials of Myers-Briggs Type Indicator assessment ( $2^{\text {nd }}$ edn). John Wiley and Sons, New York, USA, p. 210.

7. Myers IB (1998) MBTI manual: A guide to the development and use of the Myers-Briggs Type Indicator ( $3^{\text {rd }}$ edn). Consulting Psychologists Press, Palo Alto, CA, p. 420.

8. Hodges LC (1998) Students entering professional nursing: Learning style, personality type and sex-role identification. Nurse Educ Today 8(2): 68-76.

9. Li YS, Chen PS, Tsai SJ (2008) A comparison of the learning styles among different nursing programs in Taiwan: Implications for nursing education. Nurse Educ Today 28(1): 70-76.

10. Watson J (2008) Nursing: The philosophy and science of caring, (Rev. edn). University Press, Colorado, p. 336.

11. Wade GH, Kasper N (2006) Nursing students' perceptions of instructor caring: An instrument based on Watson's theory of transpersonal caring. J Nurs Educ 45(5): 162-168.

12. Ayala RA, Calvo MJ (2017) Cultural adaptation and validation of the caring behaviors assessment tool in Chile. Nurs Health Sci 19(4): 459466.

13. Chae DH, Lee CY (2014) Development and psychometric evaluation of the Korean version of the cultural competence scale for clinical nurses. Asian Nurs Res (Korean Soc Nurs Sci) 8(4): 305-312.

14. Carlson E (2013) Time, trust and reflection three aspects of precepting in clinical nursing education. Nurse Educ Prac 13(4): 237-238.

15. Faucett JM, Morgan ER, Poling TH, Johnson J (1995) MBTI type and Kohlberg's postconventional stages of moral reasoning. J Psychol Type 34:17-23.

ISSN: 2574-1241

DOI: 10.26717/BJSTR.2018.09.001803

Yuh - Shiow Li. Biomed J Sci \& Tech Res

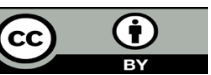

This work is licensed under Creative Commons Attribution 4.0 License

Submission Link: https://biomedres.us/submit-manuscript.php
16. Liddell DL, Davis TL (1996) The measure of moral orientation: Reliability and validity evidence. American Psycholo Assoc 37(5): 485-493.

17. Jenkins SJ, Stephens JC, Chew AL, Downs E (1992) Examination of the relationship between the Myers-Briggs Type Indicator and empathetic response. Percept Mot Skills 74: 1003-1009.

18. Jung CG (1971) Psychological types ( $9^{\text {th }}$ edn.). Princeton University Press, Minnesota, pp. 608.

19. Ou SF, Lin PF (2006) Study on the caring behavior of nursing students in a 5-year junior college. Tzu Chi Nursing Journal 5(4): 80-89.

20. Lee WL, Yang HL, Tsai SH, Tsai HY, Tsai CW (2011) Relationship between nurse caring behaviors and patient perceived caring behaviors at regional teaching hospitals in southern Taiwan. J Nurs Health Res 7(4): 286-294.

21. Pai HC, Eng CJ, Ko HL (2013) Effect of caring behavior on disposition toward critical thinking of nursing students. J Prof Nurs 29(6): 423-429.

22. Wheeler P (2001) The Myers-Briggs type indicator and applications to accounting education and research. American Accounting Education 16(1): 125-150.

23. Huszczo G, Endres M (2013) Joint effects of gender and personality on choice of happiness strategies. Europe's J Psychol 9(1): 136-149.

24. Gu XY, Hu S (2012) MBTI: New development and application. Adv Psychol Sci 20(10): 1700-1708.

25. Miao D, Huangfu E, Rosina CC, Ren J (2000) The validity analysis of the Chinese version MBTI. Acta Psychologica Sinica 32(3): 324-331.

26. Lin PF (2001) Development and psychometric evaluation of the caring behaviors scale of baccalaureate nursing student in Taiwan, Unpublished doctoral dissertation. Boston College.

27. Li YS, Chen HM, Yang BH, Liu CF (2011) An exploratory study of the relationship between age and learning styles among students in different nursing programs in Taiwan. Nurse Educ Today 31(1): 18-23.

28. Brunt BA, Kopp DJ (2007) Impact of preceptor and orientee learning styles on satisfaction: A pilot study. J Nurses Staff Dev 23(1): 36-44.

29. Australian Government Department of Health and Ageing (2008) Report on the audit of health workforce in rural and regional Australia, April 2008. Commonwealth of Australia, Canberra.

30. An Y, Kang J (2016) Relationship between organizational culture and workplace bullying among Korean nurses. Asian Nurs Res (Korean Soc Nurs Sci) 10(3): 234-239.

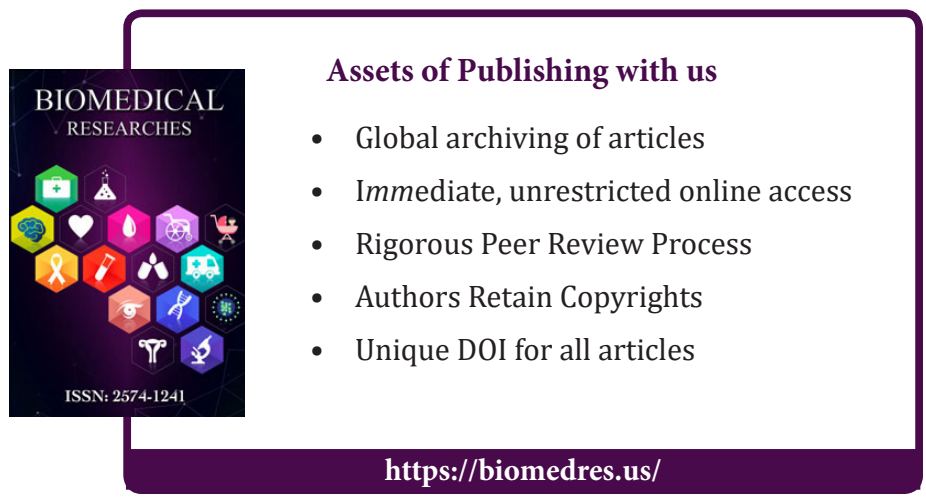

Cite this article: Yuh-Shiow Li, Wen-Pin Yu, Bao-Huan Yang, Chin-Fang Liu. Learning Styles and Caring Behaviors Among Students in Different Nursing Programs: Implications for Nursing Education. Biomed J Sci\&Tech Res 9(3)-2018. BJSTR. MS.ID.001803. 